\title{
MANIFESTACIONES REUMÁTICAS Y DERMATOLÓGICAS EN PACIENTES PAUCISINTOMÁTICOS DE COVID-19.
}

Publicado:

5 de Marzo, 2021 
Urbano Solis Cartas

Especialista en Reumatología, Máster

en Longevidad Satisfactoria.

Universidad Nacional de Chimborazo.

Escuela Superior Politécnica de

Chimborazo.

umsmwork74@gmail.com

orcid.org/0000-0003-0350-6333

Jorge Luis Valdés González

Especialista en Dermatología

Escuela Superior Politécnica de

Chimborazo

jorval1969@yohoo.es

ORCID:orcid.org/0000-0002-2661-8517
Wilmar Hernandez Perdomo

Ingeniero en Electrónica

Universidad de Las Américas

wilmar.hernendez@udla.edu.ec

orcid.org/0000-0003-4643-8377

Jose Pedro Martinez Larrarte

Especialista en Reumatología

Facultad de Ciencias Médicas Miguel

Enríquez

jpmtzl2015@gmail.com

orcid.org/0000-0003-1380-2646 
ARTíCULO CIENTÍFICO

RHEUMATIC AND

DERMATOLOGICAL

MANIFESTATIONS IN

PAUCISYMPTOMATIC COVID-19

PATIENTS. 


\section{en Español}

\section{Introducción}

Los pacientes con COVID-19 presentan un variado cortejo sintomático que incluye manifestaciones respiratorias y extra respiratorias. Dentro de este último grupo destacan las manifestaciones generales, dermatológicas y reumáticas, sin que se tenga bien claro aún el porcentaje de presentación.

\section{Objetivo}

Identificar la frecuencia con que se presentan las manifestaciones reumáticas y dermatológicas en pacientes con COVID-19.

\section{Metodología}

Se realizó una investigación descriptiva, de cohorte transversal donde la muestra estuvo constituido por 387 pacientes con diagnóstico confirmado de COVID- 19 hospitalizados en centros de salud públicos y privados de la ciudad de Riobamba, provincia Chimborazo, Ecuador en el periodo comprendido entre los meses de junio, julio y agosto del 2020. Se utilizó la observación dirigida y la revisión documental como técnicas de investigación para identificar la presencia de manifestaciones dermatológicas y reumáticas.

\section{Resultados}

Promedio de edad de 56,23 años, predominio de pacientes masculinos (55,04\%) y sin comorbilidades asociadas (36,44\%). Las manifestaciones dermatológicas se presentaron en el 33,33\% de los casos, y las maculopápulas fueron las de mayor frecuencia (53,49\%). Las manifestaciones reumáticas estuvieron en el $46,77 \%$ de los casos, y las artralgias $(51,93 \%$ ) y las mialgias $(50,28 \%)$ fueron las de mayor frecuencia.

\section{Conclusión}

Las manifestaciones dermatológicas y reumáticas se presentan con relativa frecuencia en pacientes con diagnóstico confirmado de COVID-19 como parte del cuadro clínico de la enfermedad. 


\section{en Inglés}

\section{Introduction}

Patients with COVID-19 present a varied symptomatic process that includes respiratory and extra-respiratory manifestations; within this last group, the general, dermatological, and rheumatic manifestations stand out; without the presentation percentage being clear yet.

\section{Objective}

To identify and identify the frequency with which rheumatic and dermatological manifestations occur in patients with COVID-19.

\section{Methodology}

A descriptive, cross-sectional cohort research was carried out where the sample consisted of 387 patients with a confirmed diagnosis of COVID-19 hospitalized in public and private health centers in the city of Riobamba, Chimborazo province, Ecuador in the period between the months of June, July and August 2020. Guided observation and documentary review were used as investigative techniques to identify the presence of dermatological and rheumatic manifestations.

\section{Results}

Average age of 56.23 years, predominance of male patients (55.04\%) and without associated comorbidities (36.44\%). Dermatological manifestations were present in $33.33 \%$ of cases, with maculopapules being the ones with the highest frequency of presentation (53.49\%). Rheumatic manifestations were present in $46.77 \%$ of the cases, with arthralgias (51.93\%) and myalgias (50.28\%) being the most frequent.

\section{Conclusion}

The dermatological and rheumatic manifestations appear relatively frequently in patients with a confirmed diagnosis of COVID-19 as part of the clinical picture of the disease. 


\section{en Portugués}

\section{Introdução}

Os pacientes com COVID-19 apresentam um processo sintomático variado que inclui manifestações respiratórias e extrarespiratórias. Dentro deste último grupo, destacam-se as manifestações gerais, dermatológicas e reumáticas; sem que a porcentagem de apresentação ainda esteja clara.

\section{Objetivo}

Identificar e identificar a frequência com que ocorrem as manifestações reumáticas e dermatológicas em pacientes com COVID-19.

\section{Metodologia}

Foi realizada uma pesquisa descritiva, de corte transversal, en que a amostra foi constituído por 387 pacientes com diagnóstico confirmado de COVID-19 internados em centros de saúde públicos e privados da cidade de Riobamba, província de Chimborazo, Equador no período compreendido entre os meses de Junho, julho e agosto de 2020. A observação guiada e a revisão documental foram utilizadas como técnicas investigativas para identificar a presença de manifestações dermatológicas e reumáticas.

\section{Resultados}

Idade média de 56,23 anos, predomínio de pacientes do sexo masculino (55,04\%) e sem comorbidades associadas (36,44\%). As manifestações dermatológicas estiveram presentes em 33,33\% dos casos, sendo as maculopápulas as de maior frequência de apresentação (53,49\%). As manifestações reumáticas estiveram presentes em $46,77 \%$ dos casos, sendo as artralgias $(51,93 \%)$ e as mialgias $(50,28 \%)$ as mais frequentes.

\section{Conclusão}

As manifestações dermatológicas e reumáticas aparecem com relativa frequência em pacientes com diagnóstico confirmado de COVID-19 como parte do quadro clínico da doença. 
La COVID-19 ha centrado la atención del panorama médico mundial. La rápida propagación de la enfermedad, el elevado número de contagios y muertes, ha obligado a la comunidad científica a trabajar arduamente para dar respuesta a una situación que se generó desde diciembre del 2019, y que se mantiene en la actualidad.(1)

La razón del aumento de contagios de esta enfermedad se centra en el elevado porcentaje de pacientes paucisintomáticos, quienes presentan manifestaciones clínicas que no son atribuidas a la COVID-19. Es decir, no se detecta en ellos la enfermedad en estadios iniciales, por lo que son fuente de contagio y aumentan el riesgo de presencia de complicaciones respiratorias que pueden poner en peligro la vida de los pacientes.(1,2) El principal problema de salud está en identificar determinadas manifestaciones clínicas, no respiratorias, que puedan orientar hacia la sospecha clínica de la enfermedad, incluso, en estadios iniciales, cuando aún no existe compromiso respiratorio. En muchas ocasiones el compromiso respiratorio no llega a presentarse nunca y el paciente desconoce que tiene o ha tenido la enfermedad, y continúa siendo una fuente de transmisión "asintomática".(3)

Se han descrito distintas manifestaciones extrarrespiratorias que suelen acompañar a pacientes con COVID-19. Dentro de todas ellas, las manifestaciones dermatológicas y reumáticas han sido señaladas como una de las de mayor expresión clínica en distintos estadios de la enfermedad, incluso en pacientes paucisintomáticos. Se describe que alrededor del $50 \%$ de los pacientes con COVID-19 presenta manifestaciones reumáticas y dermatológicas como parte de la expresión sistémica de esta enfermedad. (4-7)

Dentro de las manifestaciones reumáticas, los trastornos musculares como la fibromialgia, y los articulares, como la artritis reactiva, son los que mayor número de reportes abarcan; en ambos casos presentan una evolución inusual debido a una pobre respuesta a los esquemas terapéuticos habituales. $(7,8)$

En el caso de las manifestaciones dermatológicas se han descrito distintos patrones, a saber: presencia de lesiones acrales en forma de sabañones, erupciones vesiculosas, urticaria aguda, exantema maculopapular y lesiones que orientan hacia la obstrucción vascular, como es el caso del livedo reticular y otros. En la actualidad estos constituyen los principales patrones identificados y reportados. (9)

Por ello, teniendo en cuenta la situación epidemiológica mundial respecto a la COVID-19, es clave identificar elementos que permitan la sospecha clínica de la enfermedad en pacientes paucisintomáticos, y al mismo tiempo analizar los referentes internacionales que señalan la elevada presencia de manifestaciones reumáticas y dermatológicas en pacientes con COVID-19. En ese contexto, se decide realizar esta investigación con el objetivo de identificar la frecuencia con que se presentan las manifestaciones reumáticas y dermatológicas en pacientes con COVID-19 atendidos en unidades de salud de la ciudad de Riobamba, provincia de Chimborazo, Ecuador. 
Se realizó una investigación descriptiva, de cohorte transversal, la muestra estuvo constituido por 387 pacientes con diagnóstico confirmado de COVID- 19, hospitalizados en centros de salud públicos y privados de la ciudad de Riobamba, provincia Chimborazo, Ecuador, en el periodo comprendido entre los meses de junio, julio y agosto del 2020. Se incluyó la totalidad de los pacientes con diagnósticos de COVID-19.

Se utilizó la observación dirigida y la revisión documental como técnicas de investigación. La revisión documental consistió en la búsqueda de información actualizada sobre los elementos generales y específicos relacionados con el comportamiento clínico de la COVID-19, incluyendo la presencia y tipo de manifestaciones extrarrespiratorias, tales como afecciones dermatológicas y reumáticas.

También se revisaron las historias clínicas de los pacientes para identificar reportes sobre la presencia de manifestaciones reumáticas y dermatológicas, y los distintos patrones clínicos de cada una de ellas.

Para identificar presencia de fibromialgia se utilizaron los criterios diagnósticos del American College of Rheumatology (ACR), establecidos en el año 1990 y que cuentan con una sensibilidad del $88.4 \%$ y una especificidad del $81.1 \%$ (10). Para llegar al diagnóstico de artritis reactiva se tuvo en cuenta lo planteado en el 4th International Workshop on Reactive Arthritis; es decir, confirmar artritis reactiva en casos de pacientes con artritis postinfecciosa que cumplan los criterios de espondiloartropatía seronegativa (11).

Con los datos obtenidos se confeccionó una base de datos en el programa Excel que permitió homogeneizar toda la información recopilada. Se utilizó el programa estadístico SPSS, en su versión 20,5 para Windows, con el fin de realizar de forma automatizada el procesamiento de la información. Se determinaron medidas de tendencia central y de dispersión en el procesamiento de las variables cuantitativas, y frecuencias absolutas y porcentajes para las variables 
cualitativas. Los resultados fueron expresados en forma de tablas estadísticas para facilitar la comprensión de estos.

Durante el desarrollo de la investigación se cumplieron las normas y protocolos establecidos en la Declaración de Helsinki 2 para la realización de investigaciones en seres humanos (12). Cada paciente fue informado, previo a su incorporación en el estudio, de los objetivos y métodos que se iban a utilizar en la investigación. La incorporación de los pacientes fue voluntaria, después de firmar el consentimiento informado, cuya decisión quedó debidamente registrada. La investigación no generó gastos económicos para los pacientes ni sus familiares. Los resultados se utilizaron únicamente con fines investigativos y pedagógicos; todos los datos fueron tratados con absoluta confidencialidad, es decir, no se incluyeron nombres y apellidos, números de identificación, números de historias clínicas, direcciones particulares, centros de trabajo ni ningún otro elemento que pudiera ser utilizado para relevar la identidad de los pacientes. Solo en casos muy específicos se utilizaron códigos alfanuméricos. Toda la información recolectada fue resguardada con clave de seguridad a la cual solo tuvo acceso el investigador principal. Después del procesamiento de la información se eliminó la base de datos, pues se consideró que los objetivos propuestos se habían cumplido y por lo tanto no era necesario guardarla. 
Resultados

El análisis de las características generales de los pacientes confirmados con COVID-19 se observa en la tabla 1, la cual muestra un promedio de edad de 56,23 años, con predominio de pacientes entre 40 y 60 años (42,38 \%), seguido de otros con 60 años o más $(35,40$ \%). En relación con el sexo se obtuvo un predominio de pacientes masculinos (55,04 \%) en comparación con el sexo femenino.

El 36,44\% de los pacientes reportaron otras comorbilidades dentro de las que destacan la hipertensión arterial (44,68 \%) y el hipotiroidismo $(24,11 \%)$. También fueron reportadas la diabetes mellitus (21,98\%), la cardiopatía isquémica (19,86 \%) y el asma bronquial (10,64 \%). El 6,38 \% de los pacientes refirieron padecer de insuficiencia renal y solo en el 2,13 \% se recogió el antecedente de artritis reumatoide (tabla 1).

Se puede observar en la tabla 2 que el 33,33 \% de los pacientes con COVID-19 presentaron manifestaciones dermatológicas. Al analizar el tipo de manifestación dermatológica predominante se encontró como dato estadísticamente significativo que el 53,49 \% de los pacientes presentaron lesiones maculopapulares. El $32,56 \%$, lesiones acrales en forma de sabañones, y en el 29,46 \% se diagnosticaron cuadros de urticaria aguda.

En la tabla 3 se muestra que el 46,77 \% de los pacientes con diagnóstico positivo de COVID-19 presentaron, durante el curso de la enfermedad, manifestaciones reumáticas. Las manifestaciones de mayor frecuencia fueron las artralgias $(51,93 \%)$ y las mialgias (50,28\%), datos que fueron estadísticamente significativos. La artritis reactiva (38,12 \%), la fibromialgia $(29,28 \%)$ y las contracturas musculares $(17,68 \%)$ también fueron reportadas

En la tabla 4 se muestra la distribución de los pacientes teniendo en cuenta las distintas características generales estudiadas y la presencia de manifestaciones dermatológicas y reumáticas. Destaca que las manifestaciones dermatológicas se 


\section{Resultados}

presentaron con mayor frecuencia en pacientes de menor edad, y el porcentaje máximo se registró en los sujetos con COVID-19 menores de 20 años, situación que se comprobó en el 60,71\% de los casos. Sin embargo, las manifestaciones reumáticas tuvieron su mayor porcentaje de aparición en los pacientes de 60 años y más, en los que alcanzó el $60,58 \%$.

A pesar de existir un predominio de pacientes masculinos, las manifestaciones dermatológicas y reumáticas se registraron con mayor frecuencia en las pacientes femeninas. En el caso de las manifestaciones dermatológicas, se observa en el $42,52 \%$ de las mujeres y solo en el $25,82 \%$ de los hombres. Al analizar la presencia de manifestaciones reumáticas se obtuvo que el 59,19 $\%$ de las féminas presentaron este tipo de afecciones (tabla 4). Los pacientes con comorbilidades asociadas fueron los que mayor porcentaje de manifestaciones reumáticas presentaron (58,16\%); mientras que las dermatológicas se registraron con mayor frecuencia en pacientes sin comorbilidades asociadas (33,33\%) (tabla 4). 
Tabla 1: Distribución de pacientes según características generales

\begin{tabular}{|l|l|}
\hline \multirow{2}{*}{ Caracteristicas } & Muestra total 387 pacientes \\
\cline { 2 - 2 } & Frecuencia (Porcentaje \%) \\
\hline Promedio de edad (años) & 56,23 *DE 39,12 \\
\hline
\end{tabular}

Grupo de edades

\begin{tabular}{|l|l|}
\hline Menor de 20 años & $28(7.23)$ \\
\hline Entre 20 y 40 años & $58(14,99)$ \\
\hline $\begin{array}{l}\text { Mayor de } 40 \text { y menor de } 60 \\
\text { años }\end{array}$ & $164(42,38)$ \\
\hline 60 años y más & $137(35,40)$ \\
\hline
\end{tabular}

\section{Sexo}

\begin{tabular}{|l|l|}
\hline Masculino & $213(55,04)$ \\
\hline Femenino & $174(44,96)$ \\
\hline
\end{tabular}

Presencia de comorbilidades

\begin{tabular}{|l|l|}
\hline Prsencia & $141(36,44)$ \\
\hline Ausencia & $246(63,56)$ \\
\hline
\end{tabular}

Tipo de comorbilidades $n=141$ pacientes

\begin{tabular}{|l|l|}
\hline Hipertensión arterial & $63(44,68)$ \\
\hline Diabetes mellitus & $31(21,98)$ \\
\hline Asma Bronquial & $15(10,64)$ \\
\hline Cardiopatía isquémica & $28(19,86)$ \\
\hline Hipotiroidismo & $34(24,11)$ \\
\hline Insuficiencia renal & $9(6,38)$ \\
\hline Artritis reumatoide & $3(2,13)$ \\
\hline
\end{tabular}

*DE: Desviación estándar 
Tabla 2: Distribución de pacientes según presencia y tipo de manifestaciones dermatológicas.

\begin{tabular}{|l|c|c|c|}
\hline \multirow{2}{*}{$\begin{array}{l}\text { Manifestaciones } \\
\text { Dermatológicas }\end{array}$} & \multicolumn{3}{|c|}{ Muestra total 387 pacientes } \\
\cline { 2 - 4 } & Frecuencia & Porcentaje \% & *p \\
\hline Presencia & 129 & 33,33 & ---- \\
\hline Ausencia & 258 & 66,67 & ---- \\
\hline
\end{tabular}

Tipo de manifestación dermatológica n=129 pacientes

\begin{tabular}{|l|c|l|l|}
\hline Lesiones acrales & 42 & 32,56 & 0,068 \\
\hline Maculopápulas & 69 & 53,49 & 0,046 \\
\hline Urticaria aguda & 38 & 29,46 & 0,07 \\
\hline Erupción vesicular & 37 & 28,68 & 0,072 \\
\hline Livedo Reticular & 33 & 25,58 & 0,075 \\
\hline Síndrome de Raynaud & 29 & 22,48 & 0,077 \\
\hline
\end{tabular}

${ }^{*} p \leq 0,05$ 
Tabla 3: Distribución de pacientes según presencia y tipo de manifestaciones reumáticas.

\begin{tabular}{|l|c|c|c|}
\hline \multirow{2}{*}{$\begin{array}{l}\text { Manifestaciones } \\
\text { Reumáticas }\end{array}$} & \multicolumn{3}{|c|}{ Muestra total 387 pacientes } \\
\cline { 2 - 4 } & Frecuencia & Porcentaje \% & *p \\
\hline Presencia & 181 & 46,77 & ---- \\
\hline Ausencia & 206 & 53,23 & ---- \\
\hline
\end{tabular}

Tipo de manifestación reumática $n=181$ pacientes

\begin{tabular}{|l|l|l|l|}
\hline Mialgia & 91 & 50,28 & 0,049 \\
\hline Contractura muscular & 32 & 17,68 & 0,082 \\
\hline Fibromialgia & 53 & 29,28 & 0,07 \\
\hline Artralgia & 94 & 51,93 & 0,048 \\
\hline Artritis reactiva & 69 & 38,12 & 0,062 \\
\hline Síndrome oligoarticular & 17 & 9,39 & 0,09 \\
\hline
\end{tabular}

$* p \leq 0,05$ 
Tabla 4: Distribución de pacientes según características generales y presencia de manifestaciones dermatológicas y reumáticas.

\begin{tabular}{|l|c|c|c|c|c|}
\hline \multirow{2}{*}{$\begin{array}{l}\text { Características } \\
\text { Generales }\end{array}$} & \multirow{2}{*}{$\begin{array}{c}\text { Total } \\
\mathbf{n = 3 8 7}\end{array}$} & \multicolumn{4}{|c|}{ Presencia de manifestaciones } \\
\cline { 3 - 6 } & & Dermatológicas n=129 & \multicolumn{2}{c|}{ Reumáticas n=181 } \\
\hline Grupo de edades & Frecuencia & Frecuencia & Porcentaje \% & Frecuencia & Porcentaje \% \\
\hline Menor de 20 años & 28 & 17 & 60,71 & 6 & 21,43 \\
\hline Entre 20 40 años & 58 & 27 & 46,55 & 21 & 36,21 \\
\hline Mayor de 40 y menor de 60 años & 164 & 51 & 31,09 & 71 & 43,29 \\
\hline 60 años y más & 137 & 34 & 24,82 & 83 & 60,58 \\
\hline
\end{tabular}

Sexo

\begin{tabular}{|l|l|l|l|l|l|}
\hline Masculino & 213 & 55 & 25.82 & 78 & 36,62 \\
\hline Femenino & 174 & 74 & 42,52 & 103 & 59,19 \\
\hline
\end{tabular}

\section{Presencia comorbilidades}

\begin{tabular}{|l|c|c|c|c|c|}
\hline Presencia & 141 & 37 & 26,24 & 82 & 58,16 \\
\hline Ausencia & 246 & 82 & 33,33 & 99 & 40,24 \\
\hline
\end{tabular}




\section{Discusión y conclusiones}

La COVID-19 constituye una enfermedad nueva en la que, pese a las múltiples investigaciones realizadas, aún quedan muchos detalles por conocer, definir y dominar. Por ello, urge investigar acerca de los elementos que pueden minimizar los niveles de contagio de la enfermedad y realizar un diagnóstico precoz de la infección. (1)

En este sentido, es importante conocer la frecuencia con la cual se presentan manifestaciones extrarrespiratorias durante el curso clínico de la COVID-19, pues ello permitirá tener un panorama más amplio y generalizado de la enfermedad para de esa forma poder identificar, en estadios tempranos, los signos que preceden la aparición del compromiso respiratorio.

Sin duda alguna, las manifestaciones dermatológicas y reumáticas se destacan como parte de la afectación que el virus genera en el sistema inmunológico. Un elemento importante es la afectación sistémica de la enfermedad que compromete el funcionamiento de distintos órganos vitales, genera mayor compromiso hemostático y, por consiguiente, un mayor número de complicaciones que, en casos avanzados, conducen a la muerte del paciente.

La afectación muscular y articular se ha observado y reportado en otras enfermedades virales como el dengue, zika y chikungunya.(13-15) También han sido reportadas estas manifestaciones en las epidemias anteriores de SARS y MERS. $(16,17)$ Una posible explicación a este fenómeno se basa en el concepto de formación de inmunocomplejos resultantes de las toxinas liberadas por la afectación viral.(15,16) Autores como Ciaffi y otros (18) y Parisi y otros (19) describen en sus reportes cómo la presencia de mialgias y artralgias constituyen signos y síntomas frecuentemente descritos en estadios iniciales de aparición de la COVID-19.

Distintos autores han reportado la presencia de manifestaciones dermatológicas en el curso de la COVID-19. (9) Se describe que la extensión y agresividad son variables y se relacionan con los distintos patrones de afectación dermatológica. $(20,21)$ Un elemento que se destaca en esta investigación, y que ha sido señalado en otros estudios, es el relacionado con el predominio de presencia de afectación dermatológica en pacientes con menor edad. (22) Quizás esto se deba principalmente a la inmadurez de las capas dérmicas, las cuales, en edades tempranas, son más reactivas a los trastornos inmunológicos que genera la COVID-19. (23)

El patrón maculopapular fue el que se presentó con mayor frecuencia. Un resultado similar fue 
reportado en la investigación de Maqueda y otros

(9). También Bender del Busto y otros (24) describen la presencia de afectación maculopapular, de cuadros de urticaria aguda y de lesiones vesiculosas como los patrones de afectación dermatológica más frecuentes. Es importante señalar el aporte realizado por Mirza y otros (25), quienes reportan lesiones similares a los sabañones y la presencia de rash eritematoso macupapular, como las manifestaciones dermatológicas que con mayor frecuencia fueron identificadas.

Sin duda la COVID-19 se caracteriza por una elevada presencia de manifestaciones clínicas. Dentro de estas, la expresión reumática se caracteriza por afección muscular y articular, y también se registran distintos patrones de afectación dermatológica. El conocimiento de este tipo de afectaciones facilita un acercamiento rápido y seguro al diagnóstico de la enfermedad.

Las limitaciones de la presente investigación fueron dificultades relacionadas con el restringido acceso a los pacientes y a la información de la evolución de la enfermedad, así como los estrictos protocolos de bioseguridad que, en ocasiones, representaron un obstáculo para el equipo de investigadores. Otras limitaciones fueron la falta de criterios de clasificación de manifestaciones reumáticas y/o dermatológicas. Por esta razón el criterio utilizado para identificar las distintas manifestaciones reumáticas y dermatológicas se basó principalmente en los elementos semiológicos de cada una de ellas.

El equipo de investigación considera oportuno señalar que este trabajo se caracteriza por dos elementos: primero, la no inclusión dentro de los objetivos del estudio de relaciones causa efecto entre la COVID-19 y la presencia de algún tipo específico de manifestaciones reumáticas y/o dermatológicas; para ello, habría sido necesario desarrollar estudios más profundos y métodos de investigación diferentes a los utilizados. Segundo, en el desarrollo de este trabajo no se realizó modificación ni manipulación de las variables de investigación; no se realizó ninguna acción directa con los pacientes que pudiera representar algún daño, perjuicio o peligro. Por esta razón, aunque razonable, no consideramos la aprobación de un comité de ética de la investigación.

Se concluye que las manifestaciones dermatológicas (Foto1) y reumáticas (Foto 2) se presentan con relativa frecuencia en pacientes con diagnóstico confirmado de COVID-19 como parte del cuadro clínico de la enfermedad. Las manifestaciones dermatológicas se presentan con mayor frecuencia en pacientes menores de 40 años, de sexo femenino y sin presencia de comorbilidades. Las manifestaciones reumáticas son más frecuentes de encontrar en pacientes mayores de 40 años de edad, femeninas y con presencia de comorbilidades asociadas. 


\section{Foto 1: Ejemplo Manifestación Dermatológica}

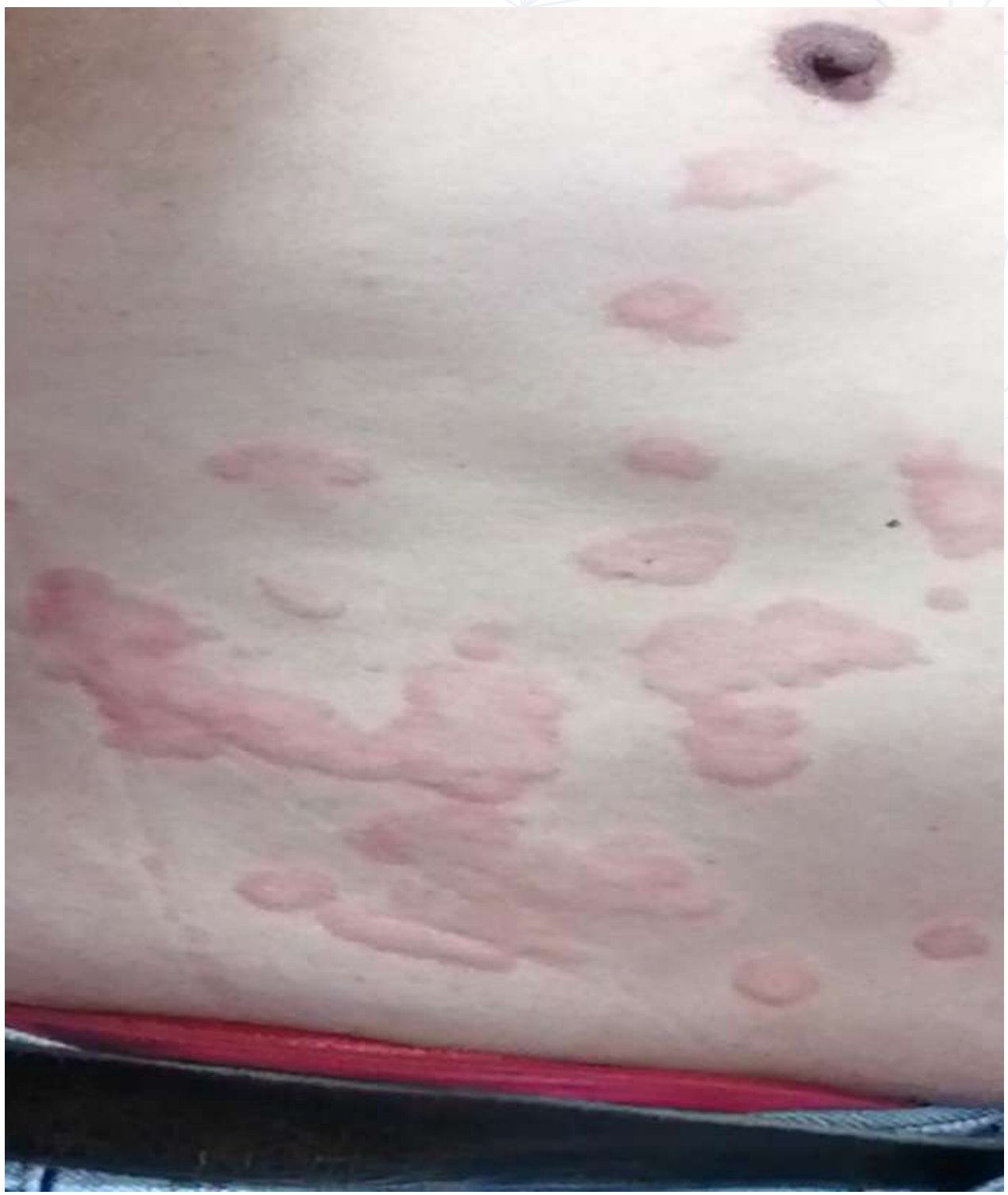

Lesiones dermatológicas consistentes con patrón de afectación urticariforme en un caso con diagnóstico confirmado de COVID-19 
Foto 2: Ejemplo Manifestación Articular

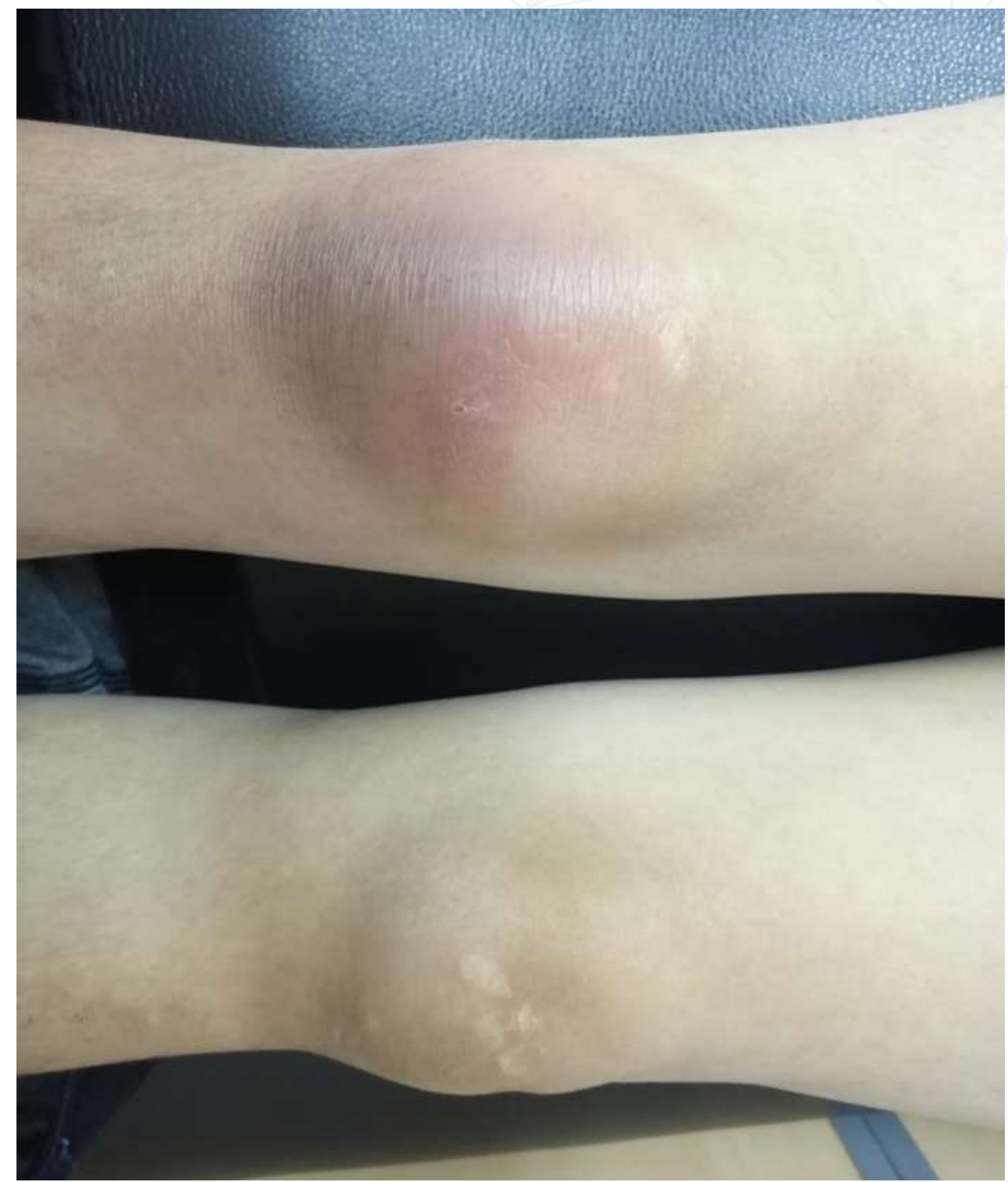

Cuadro inflamatorio monoarticular consistente en la presencia de inflamación y rubor a nivel de rodilla derecha en un paciente con diagnóstico confirmado de COVID-19 
F in a n c i c ión

No se recibió financiamiento para la realización de esta investigación. Los gastos fueron sufragados por los autores. 


\section{Referencias}

1. Solis-Cartas U, Martínez-Larrarte J. Opciones terapéuticas al síndrome de liberación de citocinas en pacientes con la COVID-19. Revista Cubana de Medicina Militar [Internet], 2020 [citado 2020 Sep 13];49(3):e0200783. Disponible en: http://wwW.revmedmilitar.sld.cu/index.php/mil/article/view/783

2. Solis-Cartas U. Coronavirus y enfermedades reumáticas, suposiciones, mitos y realidades. Rev Cubana de Reumatolo [Internet]. 2020 [citado 2020 Sep 16];22(2):[aprox. 5 p.]. Disponible en: http://revreumatologia.sld.cu/index.php/reumatologia/article/view/791

3. Chia-Proenza D, Gómez-Conde SY, del Toro-Ravelo LM. Acercamiento a la COVID-19 desde una perspectiva pediátrica. Rev Cubana de Reumatolo [Internet]. 2020 [citado 2020 Sep 17];22(2):[aprox. 6 p.]. Disponible en: http://www.revreumatologia.sld.cu/index.php/reumatologia/article/view/830

4. Reyes-Llerena GA, Guibert-Toledano ZM, Hernández-Martínez AA, Otero-González ZA, Bicet-Dorzón $E$, et al. Las enfermedades autoinmune-reumáticas en la epidemia por SAR-COV-2; COVID-19. Estrategias en su manejo y actualización desde la visión de la sociedad cubana de reumatología y su grupo nacional. Rev Cubana de Reumatolo [Internet]. 2020 [citado 2020 Sep 12];22(3):[aprox. 8 p.]. Disponible en: http://www.revreumatologia.sld.cu/index.php/reumatologia/article/view/852
5. Gómez-Conde SY, Chia-Proenza D, Del Toro-Ravelo LM. Revisión de las recomendaciones para enfermos reumáticos y la COVID-19. Propuesta para los pacientes pediátricos cubanos con enfermedad reumática y sus familiares. Rev Cubana de Reumatolo [Internet]. 2020 [citado 2020 Sep 12];22(2):[aprox. 6 p.]. Disponible en: http://www.revreumatologia.sld.cu/index.php/reumatologia/article/view/831

6. Tufan ZK, Kayaaslan B. Crushing the curve, the role of national and international institutions and policy makers in COVID-19 pandemic. Turk J Med Sci. 2020; 50(SI-1): 495- 508.

7. Cai S, Sun W, Li M. A complex COVID-19 case with rheumatoid arthritis treated with tocilizumab. Clin Rheumatol 2020; (39):2797-2802. Disponible en: https://doi.org/10.1007/s10067-020-05234-w

8. Caso F, Costa L, Ruscitti P, Navarini L, Del Puente A, Giacomelli R. Could Sars-coronavirus-2 trigger autoimmune and/or autoinflammatory mechanisms in genetically predisposed subjects? Autoimmunity Reviews [Internet]. 2020 [citado 2020 Sep 14];19(5):102524. Disponible en: https://www.sciencedirect. com / s cience/art i cle/pii/S1568997220300781

9.- Maqueda-Zamora G, Sierra-Santos L, Sierra-Santos E, Martínez- Ballester JF. Manifestaciones dermatológicas de la infección por COVID-19 en Pediatría. Rev Clin Med Fam [Internet]. 2020[citado 
2020 Sep 16];13(2):166-70. Disponible en: http://scielo.isciii.es/scielo.php?script =sci_arttext\&pid=S1699-695X2020000200166\&lng=es

10. Wolfe F, Smythe HA, Yunus MB, Bennett RM, Bombardier C, Goldenberg DL, Tugwell P, Campbell SM, Abeles M, Clark P, et al. The American College of Rheumatology 1990 Criteria for the Classification of Fibromyalgia. Report of the Multicenter Criteria Committee. Arthritis Rheum. 1990 Feb;33(2):160-72. doi: 10.1002/art.1780330203. PMID: 2306288

11. Sieper J, Braun J, Kingsley GH. Report on the Fourth International Workshop on Reactive Arthritis. Arthritis Rheum. 2000 Apr;43(4):720-34. doi: 10.1002/1529-0131(200004)43:4<720::AID-ANR2>3 .0.CO;2-8. PMID: 10765916.

12. Declaration of Helsinki of the World Medical Association - Ethical principles for medical research involving human subjects. Disponible en https://www.wma.net/es/policies-post/declara$c i o n-d e-h$ e l s i $n$ ki-de-la-amm-principios-eticos-para-las-investigacio nes-medicas-en-seres-humanos/ ).

13. Espinoza M. Aspectos clínicos de la infección por el virus Zika. An. Fac. med. [Internet]. 2017[citado 2020 Sep 15];78(1):79-82. Disponible en: http://www.scielo.org.pe/scielo.php?script=sci_arttext\&pid=\$1025-55832017000100013\&lng=es

14. rredondo-Bruce $A E$, González-De la Cruz $Y$, Martínez-Chávez S, Arredondo-Rubido AE. Manifestaciones tardías del dengue grave en pacientes de la provincia de Camagüey. Rev. electron. Zoilo [Internet]. 2016 [citado 2020 Sep 16];41(11):[aprox. 8 p.]. Disponible en: http://www.revzoilomarinello.sld.cu/index.php/zmv/article/view/877
15. Turtos-Díaz LM, Ricardo-Santiesteban ON, Clemente-Ricardo O, Navarro-Arrieta S, Hernández-Cruz. Chikungunya: Una manifestación atípica en la piel. Reporte de un caso. ccm [Internet]. 2019[citado 2020 Sep 17];23(1):288-96. Disponible en: http://scielo.sld.cu/scielo.php?script=sci_arttext\&pid=S1560-43812019000100288\&lng=es

16. Piamo Morales AJ, García-Rojas M. SARS-CoV, MERS-CoV y SARS-CoV-2: lo que se sabe de estos coronavirus epidémicos. Rev Panorama. Cuba y Salud [Internet]. 2020 [citado 2020 Sep 16 ];15(3):64-75. Disponible en: http://www.revpanorama.sld.cu/index.php/rpan/article/view/

17. Góngora-Gómez O, Gámez-Leyva LR. Manifestaciones extrapulmonares de la infección por el nuevo Coronavirus SARS-CoV-2. Rev haban cienc méd [Internet]. 2020 [citado 2020 Sep 19] ; 19( Suppl 1 ): e3378. Disponible en: http://scielo.sld.cu/scieIo. php? script =sci_arttext\&pid=S1729519X2020000400006\&lng=es

18. Solis-Cartas U, Calvopiá-Bejarano SJ, Nuñez-Sánchez BL, Yartú-Couceiro R. Relación entre adiposidad corporal y presión arterial en niños y adolescentes con enfermedades reumáticas. Rev Cuba Reumatol [Internet]. 2019 [citado 2020 Sep 16]; 21(1):e51. Disponible en: http://scielo.sld.cu/scieI o. ph p ? s c r i p t = s c i - a r t t e x t\&pid=S1817-59962019000100002\&lng=es

19. Solis-Cartas U, Ríos-Guarango PA, López-Proaño GF, Yartú-Couceiro R, Nuñez-Sánchez BL, AriasPastor $\mathrm{FH}$ et al. Uso de la medicina natural en el tratamiento de la artritis reumatoide. Rev Cuba Reumatol [Internet]. 2019[citado 2020 Sep 17];21(3):e61. Disponible en: http://scielo.sld.cu/scielo.php? script = s ci - art text\&pid=S1817-59962019000300004\&lng=es 
20. Ciaffi J, Meliconi R, Ruscitti P, Berardicurti O, Giacomelli R, Ursini F. Rheumatic manifestations of COVID-19: a systematic review and meta-analysis. BMC Rheumatology [Internet]. 2020 [citado 2020 Sep 15]; 4 (65): 2-15. Disponible en: https://bmcrheumatol. biomed central. com/art i cles/10.1186/s41927-

020-00165-0

21. Parisi S, Borreli R, Bianchi S, Fusano E. Viral arthritis and COVID-19. The Lancet Rheumatology [Internet]. 2020 [citado 2020 Sep 16]; 2 (11): e65-67. Disponible en: https://doi.org/10.1016/S2665-

9913(20)30348-9

22. Granados-Campos L, Broche-del Pino L, Pérez-Leal L, López-Rodríguez VM. Manifestaciones cutáneas en pacientes pediátricos infectados por el coronavirus SARS-CoV-2. Rev Cubana Pediatr [Internet]. 2020 [citado 2020 Sep 16];92(Suppl1): e1171. Disponible en: http://scielo.sld.cu/scieI o. ph p ? s c r i p t = s c i - art t ex t\&pid=S0034-75312020000500009\&lng=es

23. Macías Ma. A, Zapata E, González Ma. C, Fuenzalida H, Honeyman J, Chávez F, et al. Recomendaciones Rama de Dermatología Pediátrica, Sociedad Chilena de Pediatría. Pandemia COVID-19. Rev. chil. pediatr. [Internet]. [citado 2020 Sep 16]. Disponible en:

https://scielo.conicyt.cl/scieI o.php ? s c r i p t = s ci_art t e x t\&pid=S0370-41062020005001005\&lng=es

24. Bender-del Busto J, León-Castellón R, Mendieta-Pedroso M, Rodríguez-Labrada R, Velázquez-Pérez L. Infección por el SARS-CoV-2: de los mecanismos neuroinvasivos a las manifestaciones neurológicas. Anales de la Academia de Ciencias de Cuba [Internet]. 2020 [citado 2020 Sep 16];10(2):[aprox. 7 p.]. Disponible en: http://www.revistaccuba.sld.cu/index.php/revacc/arti- cle/view/855

25. Mirza F, Malin A, Omer S, Sethi A. Dermatologic manifestations of COVID-19: a comprehensive systematic review. International Journal of Dermatology [Internet. 2020; [citado 2020 Sep 14]1-33. Disponible en: https://doi.org/10.1111/ijd.15168
(1)

\section{.}


GLOBAL RHEUMATOLOGY 